\title{
Age, Sex, and Regional Differences in the Effect of COVID-19 Pandemic on Objective Physical Activity in Japan: A 2-Year Nationwide Longitudinal Study
}

\author{
Y. Yamada ${ }^{1}$, T. Yoshida ${ }^{1}$, T. Nakagata ${ }^{1}, H$. Nanri $^{1}$, M. Miyachi ${ }^{1,2}$

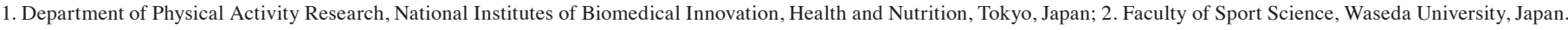 \\ Corresponding Author: Yosuke Yamada, PhD, 1-23-1 Toyama, Shinjuku-ku, Tokyo, 162-8636 Japan, yamaday@nibiohn.go.jp
}

Key words: Physical activity, urban, rural, step count, COVID-19 pandemic.

\section{Dear Editor,}

$\Lambda$ lthough more than a year has passed since the first detection of coronavirus disease (COVID19) in Wuhan, China, it remains pandemic. Many governments have advocated for social distancing measures with varying degrees of enforcement, ranging from unforced recommendations to business closures and city lockdowns. This induced sudden and fundamental changes in the lifestyles of populations. A study of the daily step counts from January 19 to June 1, 2020, measured using smartphones, found that step count decreased from March to the end of May 2020 worldwide (1). Online surveys using International Physical
Activity Questionnaire shows physical activity deceased in April 2020 and recovered in June 2020 (2, 3). Because physical activity and daily step counts are determinants of health (4), they should be monitored and explored in depth. Physical activity varies considerably according to gender, age group, region, and season; therefore, data on physical activity before the COVID-19 pandemic are needed for reference. .

We assessed the age, gender, and regional differences in the effects of the COVID-19 pandemic on daily step count in Japan using activity intensity-dependent triaxial accelerometers, which used an algorithm that had been validated using a metabolic chamber and the doubly-labeled water method (5).

In this longitudinal study, we used individual data from January 1, 2019, to January 1, 2021, collected from users of triaxial-accelerometer-based physical activity monitors (Panasonic, Osaka, Japan) (5). A total of 628,111 observationdays were provided by 1,167 unique users nationwide in

Figure 1. Step counts according to month in 2019 and 2020

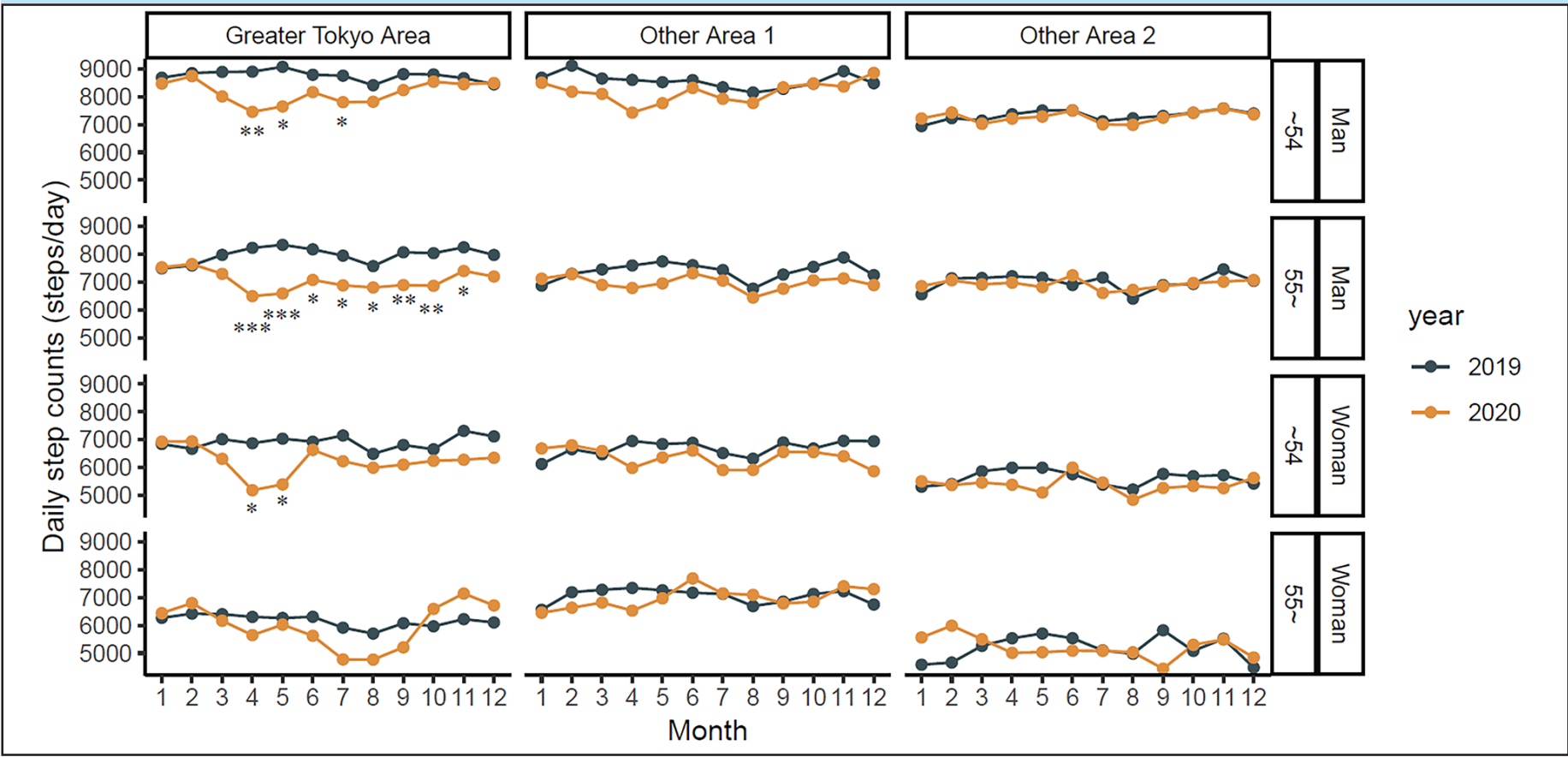

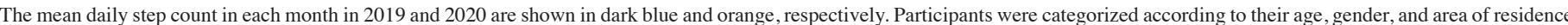

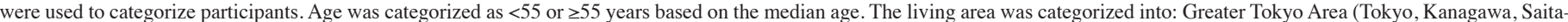

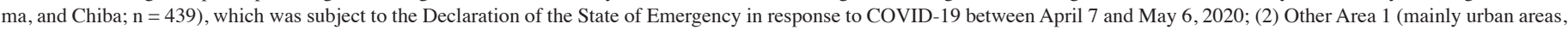

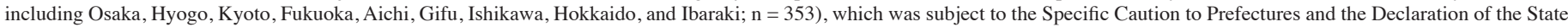

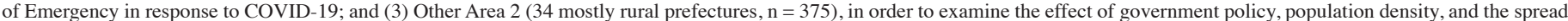

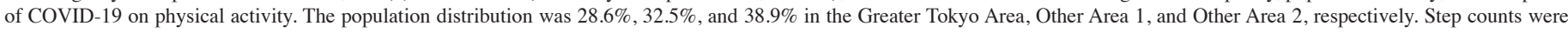

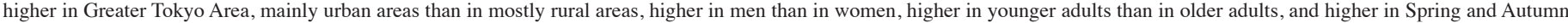
than winter and mid-summer in 2019 . Step counts significantly decreased in 2020 in Greater Tokyo area. $* \mathrm{P}<0.05$, $* * \mathrm{P}<0.01, * * * \mathrm{P}<0.001$, significantly lower than 2019 . 
Figure 2. Physical activity energy expenditure (AEE) according to month in 2019 and 2020

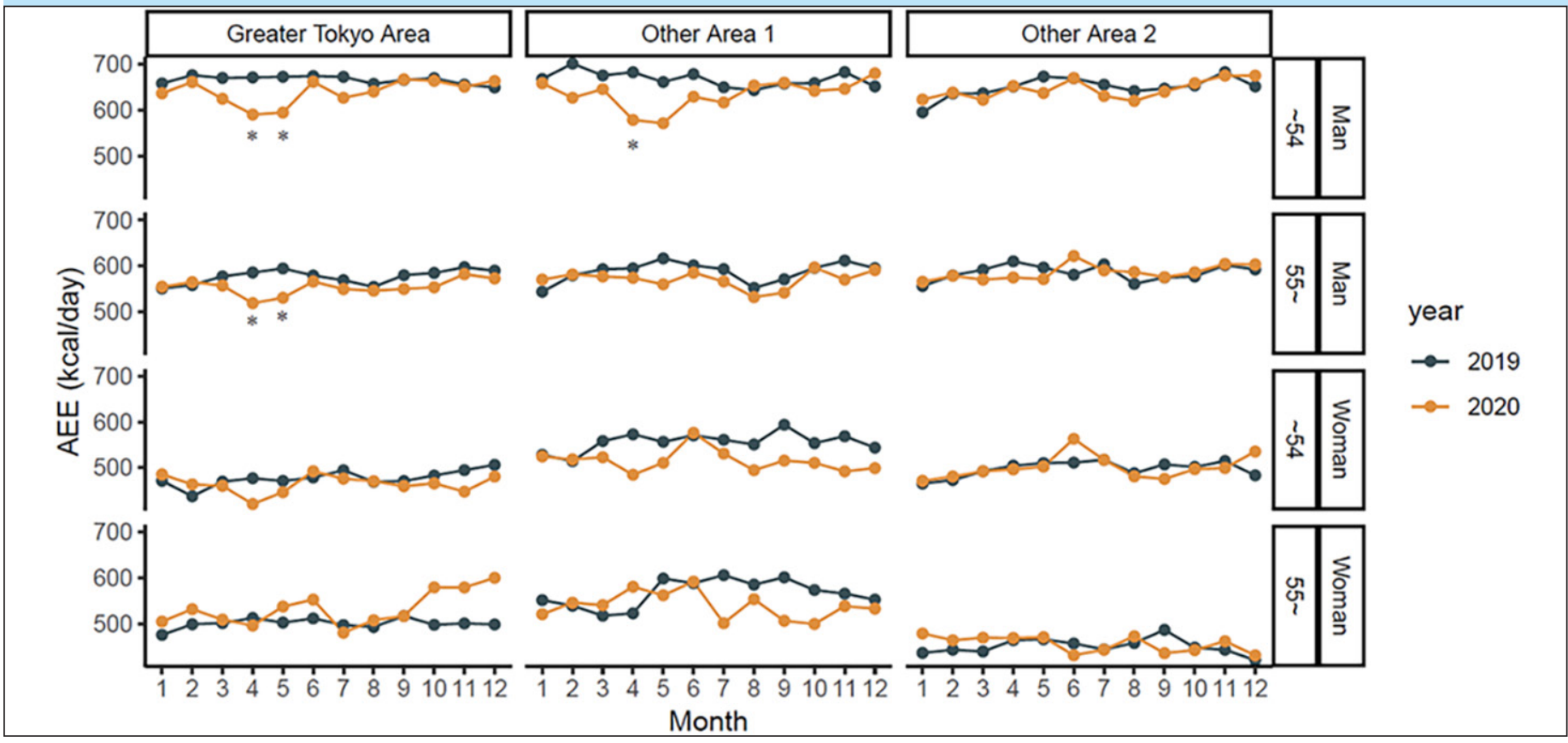

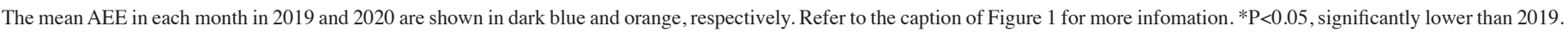

Japan. The study was conducted with approval by the ethics committees of National Institutes of Biomedical Innovation, Health and Nutrition (No. 202). All participants read an explanation and indicated their understanding before providing informed consent.

Seasonal variation, gender, and age group differences were observed in the step counts and physical activity energy expenditure (AEE) in 2019 (Figures 1 and 2). Compared with 2019, a significant decline in the step count was observed in March 2020 in younger and older men, and younger women, but not in older women in the Greater Tokyo area. After the Declaration of a State of Emergency on April 7, 2020, and implementation of regional restrictions, the step count decreased further by up to 1800 steps/day in April and May 2020 in younger and older men, and younger women, but not in older women. The step count gradually increased after the lifting of regional orders. By October 2020, when the government included Tokyo prefecture into their economic revitalization strategies named "GO TO TRAVEL and GO TO EAT," the step count had returned to the 2019 baseline levels in younger men and older women, but not in older men and younger women. In rural areas, the step count and AEE in 2020 were similar to those in 2019.

Japan tended to have low COVID-19 infection rates in 2020 and did not have mandatory lockdowns, but residents of the Greater Tokyo Area and urban areas still showed large decreases in their step counts and AEE during the period immediately after COVID-19 was declared a global pandemic. Responses to the COVID-19 pandemic varied according to the region, age, and gender, which is probably attributable to a combination of changes to activities of daily living (e.g., commuting and shopping) and exercise habits (e.g., walking and exercising). The observed regional variation in physical activity is also likely to have been influenced by environmental differences and differences in the use of cars and public transportation between regions. Limitations of this study include sampling bias due to convenience sampling and variability of compliance in wearing devices. This study showed clear differences in the impact of the COVID-19 pandemic on physical activity within regions according to age and gender. Social distancing measures and government requests or orders decreased overall physical activity, which is an important determinant of health. The long-term effect of decreased physical activity should be monitored.

Author contributions: YY, TY, TN, HN, MM study design and concept; YY analyzed data and wrote the draft; TY, TN, HN, MM revised the draft substantially; All authors read and approved the final manuscript.

Conflicts of interest: The authors declare that there are no conflicts of interest.

\section{References}

1. Tison GH, Avram R, Kuhar P, et al. Worldwide Effect of COVID-19 on Physical Activity: A Descriptive Study. Ann Intern Med. 2020;173(9):767-770, doi: 10.7326/ M20-2665.

2. Yamada M, Kimura Y, Ishiyama D, et al. Letter to the Editor: Recovery of Physical Activity among Older Japanese Adults since the First Wave of the COVID-19 Pandemic. J Nutr Health Aging. 2020;24(9):1036-1037, doi: 10.1007/s12603-0201516-z.

3. Yamada M, Kimura Y, Ishiyama D, et al. Effect of the COVID-19 Epidemic on Physical Activity in Community-Dwelling Older Adults in Japan: A Cross-Sectional Online Survey. J Nutr Health Aging. 2020;24(9):948-950, doi: 10.1007/s12603-0201516-z.

4. Saint-Maurice PF, Troiano RP, Bassett DR, Jr., et al. Association of Daily Step Count and Step Intensity With Mortality Among US Adults. JAMA. 2020;323(12):11511160, doi: 10.1001/jama.2020.1382.

5. Murakami H, Kawakami R, Nakae S, et al. Accuracy of Wearable Devices for Estimating Total Energy Expenditure: Comparison With Metabolic Chamber and Doubly Labeled Water Method. JAMA Intern Med. 2016;176(5):702-703, doi: 10.1001/jama.2020.1382.

How to cite this article: Y. Yamada, T. Yoshida, T. Nakagata, et al. Age, Sex, and Regional Differences in the Effect of COVID-19 Pandemic on Objective Physical Activity in Japan: A 2-Year Nationwide Longitudinal Study. J Nutr Health Aging. 2021;25(8):10321033; http://dx.doi.org/10.1007/s12603-021-1662-y 